\title{
Occult stage IIIA-N2 patients have excellent overall survival with initial surgery
}

\author{
Min P. Kim ${ }^{1,2}$, Arlene M. Correa ${ }^{1}$, Wayne L. Hofstetter ${ }^{1}$, Reza J. Mehran ${ }^{1}$, David C. Rice ${ }^{1}$, Jack A. Roth ${ }^{1}$, \\ Ara A. Vaporciyan ${ }^{1}$, Garrett L. Walsh ${ }^{1}$, Jeremy J. Erasmus ${ }^{3}$, Stephen G. Swisher ${ }^{1}$ \\ ${ }^{1}$ Department of Thoracic and Cardiovascular Surgery, The University of Texas MD Anderson Cancer Center, Houston, Texas, USA; ${ }^{2}$ Division of \\ Thoracic Surgery, Department of Surgery, Houston Methodist, Houston, Texas, USA; ${ }^{3}$ Department of Diagnostic Radiology, The University of \\ Texas MD Anderson Cancer Center, Houston, Texas, USA \\ Contributions: (I) Conception and design: All authors; (II) Administrative support: MP Kim, SG Swisher; (III) Provision of study materials or patients: \\ All authors; (IV) Collection and assembly of data: MP Kim, AM Correa, SG Swisher; (V) Data analysis and interpretation: MP Kim, AM Correa, \\ SG Swisher; (VI) Manuscript writing: All authors; (VII) Final approval of manuscript: All authors. \\ Correspondence to: Stephen G. Swisher, MD. Division of Surgery, Department of Thoracic and Cardiovascular Surgery, The University of Texas MD \\ Anderson Cancer Center, 1400 Pressler St, FCT 10.5040, Houston, Texas 77030, USA. Email: sswisher@mdanderson.org.
}

Background: Patients may be found to have stage IIIA-N2 at the final pathology after the initial surgery. We want to determine the survival rate in this unique group of patients.

Methods: We reviewed all patients who underwent surgical resection for lung cancer from 2000 to 2011 who had pathologic stage N2 without induction therapy. We determined the clinicopathologic characteristics and survival rate in this unique group of patients.

Results: A total of 101 patients met the inclusion criteria. The average age of the group was 65 years old with 53 (53\%) females. The chest computed tomography (CT) scans showed 30 patients (30\%) with mediastinal lymphadenopathy $(>1 \mathrm{~cm})$ and $13(13 \%)$ with multistation disease. The positron emission tomography-computed tomography (PET-CT) showed 24 patients (24\%) with N2 positive uptake. Invasive mediastinal staging prior to surgery occurred in 43 patients $(43 \%)$. Eighty-four patients underwent a lobectomy (83\%), 7 with bilobectomy (7\%), and 10 with pneumonectomy (10\%). The most common pathology was adenocarcinoma with 73 patients $(72 \%)$ and the second most common was squamous cell carcinoma with 22 patients (22\%). Most of the patients completed the adjuvant chemoradiation therapy (86\%). The 5 -year survival rate was $48 \%$ and the 10 -year survival rate was $24 \%$.

Conclusions: Pathologic stage IIIA-N2 non-small cell lung cancer (NSCLC) is a heterogeneous disease process with a very small group of patients undergoing initial surgery. Patients with occult stage IIIA-N2 who undergo initial surgery have an excellent overall survival rate.

Keywords: Lung cancer; stage IIIA-N2; surgery; survival

Submitted Jun 18, 2018. Accepted for publication Oct 18, 2018.

doi: $10.21037 /$ jtd.2018.10.94

View this article at: http://dx.doi.org/10.21037/jtd.2018.10.94

\section{Introduction}

Lung cancer treatment and survival depend on the patient's clinical stage. Among all of the different stages, patients with stage IIIA-N2 disease face the most controversial treatment path because stage IIIA-N2 disease is a heterogeneous disease process with some patients presenting with microscopic tumors in the mediastinal lymph node while other patients have bulky multistation disease. Even though patients with this stage have different amounts of disease burden in the mediastinum, we provide a uniform treatment to this group of patients. Roth et al. and Roswell et al. have shown that patients who underwent induction chemotherapy followed by surgery 
had significantly improved survival compared to patients who had surgery alone. These studies from the 1990s have shaped our standard treatment plan for patients with stage IIIA-N2 disease (1,2). Roth et al. (3) and Rosell et al. (4) have shown significant improved survival with induction chemotherapy for stage IIIA disease compared to surgery alone. Other studies have shown the benefits of induction therapy but they all include a mixed group of patients spanning from stage IB to IIIA, thus the overall survival in this group of patients does not apply to our current study (5-8). Patients with concurrent chemoradiation therapy followed by surgery for stage IIIA showed a median survival rate of 13 months in one study (9) and 50.4 months in another (10).

All of these studies showed a clear benefit in providing induction therapy for patients with $\mathrm{N} 2$ disease. However, all of these randomized patients had N2 disease that was detected on computed tomography $(\mathrm{CT})>1 \mathrm{~cm}$ and was confirmed on mediastinoscopy. None of these patients underwent positron emission tomography-computed tomography (PET-CT). Since these studies have been performed, we have improved our ability to stage the mediastinum with PET-CT, which has a higher sensitivity and specificity compared to CT scans alone. In addition to mediastinoscopy, we have endobronchial ultrasound (EBUS) to help obtain confirmatory tissue diagnosis.

In the era of improved mediastinal staging modalities, we still have patients who undergo initial surgery and are found to have N2 disease at the final pathology (11). This group of patients have what we call "occult N2" disease because they either have no PET or CT criteria for mediastinal metastasis or have PET or CT positive N2 lymph nodes with either EBUS and/or mediastinoscopy that did not yield a diagnosis of metastatic disease. The rate of occult N2 disease is $4-6 \%$ for $\mathrm{T} 1$ tumors and $9-10 \%$ for $\mathrm{T} 2$ tumors with higher rates in patients with larger $\mathrm{T}$ size, positive $\mathrm{N} 1$ and central tumors $(12,13)$. In this study, we want to determine the clinicopathologic characteristics and survival for patients with occult N2 disease, and determine if any factors contribute to their survival.

\section{Methods}

The University of Texas MD Anderson Cancer Center (MDACC) Institutional Review Board approved this retrospective study (PA15-0619). We identified patients who underwent pulmonary resection (lobectomy, bilobectomy and pneumonectomy) for primary lung cancer at MD Anderson Cancer Center (MDACC) between 2000 and 2013. We then determined those patients who had pathologic stage IIIA-N2 disease who underwent surgery as an initial therapy according to the Seventh Edition of American Joint Committee on Cancer (AJCC) staging guidelines. Our inclusion criteria for the study was that the patient had to have preoperative 2-deoxy-2-(18F) fluoro$\mathrm{D}$-glucose positron emission tomography (FDG-PET) or FDG-PET/CT and CT scan for non-invasive staging of the mediastinum within 60 days of the time of surgery. We excluded those patients with carcinoid tumors. The standard protocol for invasive mediastinal staging was those patients who had any CT or FDG-PET evidence, or large tumor or central tumor underwent mediastinal staging. If the EBUS was negative but still high clinical suspicion, patient underwent mediastinoscopy.

The prospective database and the patient's medical records were used to collect demographic information, comorbidity, type of operation, pathologic lung stage, histologic evaluation, adjuvant chemotherapy, adjuvant radiation therapy, follow-up and mortality data. The CT or report was reviewed to determine whether the mediastinal lymph node met the CT criteria for lymphadenopathy $(>1 \mathrm{~cm})$ and PET-CT or its report was reviewed for standardized uptake value (SUV) of the mediastinal lymph node and primary tumor.

We determined the clinicopathologic and radiological characteristics of patients who met the inclusion and exclusion criteria. We determined the mean and standard deviation for continuous variables and determined the percentage for categorical variables. The overall survival curve was obtained using the Kaplan-Meier method. We then performed univariable Cox regression analysis for overall survival rates, and those covariates with $P$ value of less than 0.25 were entered into a multivariable analysis. Stepwise Wald backward elimination was used in multivariable Cox regression analysis to identify variables predictive of overall survival in the group. The statistical significance was defined as a $\mathrm{p}$ value of less than 0.05 . We used SPSS 21 software (IBM Corp, Armonk, NY, USA) to perform the statistical analysis.

\section{Results}

There were 2,817 patients who underwent lobectomy, bi-lobectomy or pneumonectomy for primary lung cancer between 2000 and 2013. There were 101 patients $(3.6 \%)$ who met the inclusion and exclusion criteria. The 
Table 1 Clinicopathologic characteristics

\begin{tabular}{|c|c|}
\hline Characteristic & Total $(n=101, \%)$ \\
\hline Age (mean \pm STDEV) & $65 \pm 11$ \\
\hline Male & $48[48]$ \\
\hline \multicolumn{2}{|l|}{ Co-morbidity } \\
\hline COPD & 13 [13] \\
\hline Diabetes & 12 [12] \\
\hline Renal insufficiency & $1[1]$ \\
\hline Cerebral vascular accident & 3 [3] \\
\hline Myocardial infarction & $3[3]$ \\
\hline Arrhythmia & $6[6]$ \\
\hline CAD & $15[15]$ \\
\hline Hypertension & 38 [38] \\
\hline Cardiovascular disease & $3[3]$ \\
\hline \multicolumn{2}{|l|}{ Smoking history } \\
\hline Any & $80[79]$ \\
\hline \multicolumn{2}{|l|}{ CT imaging } \\
\hline Multistation & $13[13]$ \\
\hline Bulky on CT (>1 cm) & $30[30]$ \\
\hline \multicolumn{2}{|l|}{ Imaging } \\
\hline CT positive only & 18 [18] \\
\hline PET positive only & $12[12]$ \\
\hline CT and PET positive & $12[12]$ \\
\hline Any positive & $42[42]$ \\
\hline CT and PET negative & $59[58]$ \\
\hline \multicolumn{2}{|l|}{ Mediastinal staging } \\
\hline EBUS & $14[14]$ \\
\hline Mediastinoscopy & 29 [29] \\
\hline None & $58[57]$ \\
\hline \multicolumn{2}{|l|}{ Type of surgery } \\
\hline Lobectomy & 84 [83] \\
\hline Bi-lobectomy & $7[7]$ \\
\hline Pneumonectomy & $10[10]$ \\
\hline
\end{tabular}

Table 1 (continued)

average age of the group was 65 years old with $53(53 \%)$ females (Table 1). There were 36 patients who had FDGPET and 65 patients who had FDG-PET/CT. The CT
Table 1 (continued)

\begin{tabular}{lc}
\hline Characteristic & Total $(\mathrm{n}=101, \%)$ \\
\hline Pathologic T stage & $20[20]$ \\
T1a & $15[15]$ \\
T1b & $44[44]$ \\
T2a & $12[12]$ \\
T2b & $10[10]$ \\
T3 & \\
Location of N2 disease & $72[71]$ \\
Central N2 $[2,4,7]$ & $29[29]$ \\
Peripheral N2 [5, 6, 9] & \\
Pathology & $73[72]$ \\
Adenocarcinoma & $22[22]$ \\
Squamous cell carcinoma & $4[4]$ \\
Adenosquamous & $2[2]$ \\
Large cell & $84[86]$ \\
Post-op chemo ( $\mathrm{n}=98)$ & $84[87]$ \\
Post-op rad ( $\mathrm{n}=97)$ & \\
\hline
\end{tabular}

CT positive or Bulky CT lymph node, any mediastinal lymph node $>1 \mathrm{~cm}$. PET positive, positive lymph node on report. STDEV, standard deviation; COPD, chronic obstructive pulmonary disease; CAD, coronary artery disease; CT, computed tomography; PET, positron emission tomography; EBUS, endobronchial ultrasound.

of the chest showed 30 patients $(30 \%)$ with mediastinal lymphadenopathy (>1 cm) and $13(13 \%)$ with multistation disease. The PET-CT showed 24 (24\%) with N2 positive uptake. Among 101 patients, 43 (43\%) patients had either EBUS or mediastinoscopy for invasive staging that showed negative metastatic disease to the mediastinal lymph node. Eighty-four patients underwent surgery with lobectomy (83\%), 7 with bilobectomy (7\%), and 10 with pneumonectomy (10\%). The most common pathology was adenocarcinoma with 73 patients (72\%) and the second most common was squamous cell carcinoma with 22 patients $(22 \%)$. The final pathology showed 72 patients (71\%) with positive lymph nodes in station 2, 4 or 7 and rest of the patients with positive lymph nodes in station 5, 6 or 9. We had data for 98 patients for completion of adjuvant chemotherapy and 97 patients for completion of adjuvant radiation therapy. Among these patients, $86 \%$ and 


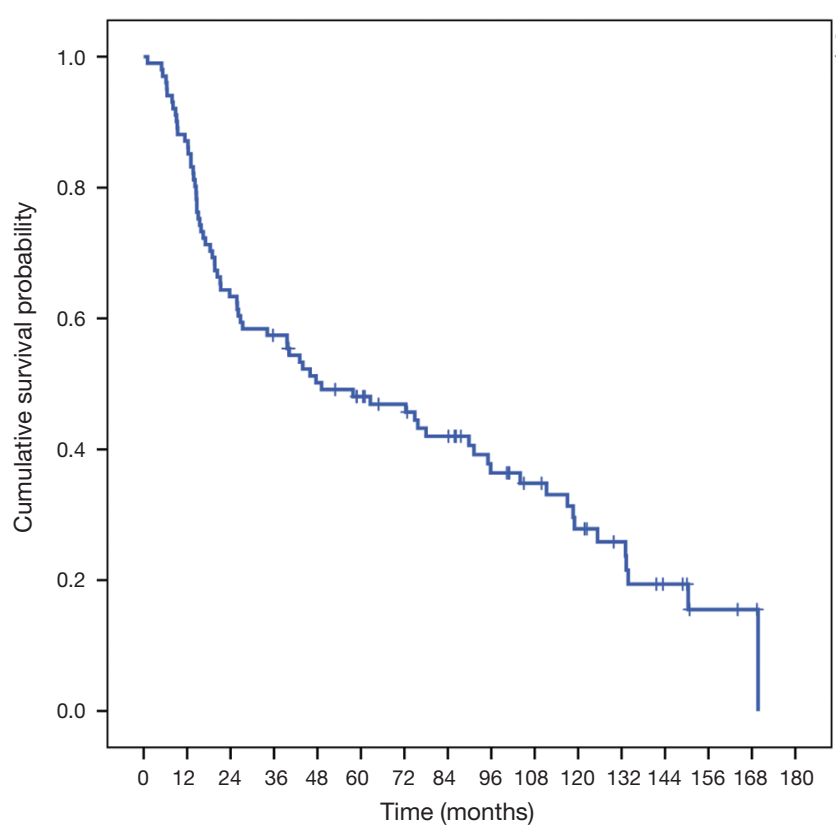

Figure 1 Overall survival. Kaplan-Meier curve showing the overall survival. The median survival for the entire group was 49 months and the 5 -year survival rate was $48 \%$ and 10 -year survival rate was $24 \%$.
$87 \%$ completed the adjuvant therapy respectively.

The median survival for the entire group was 49 months and the 5-year survival rate were $48 \%$ and 10 -year survival rate was $24 \%$ (Figure 1). For patients with CT and PET negative preoperative imaging, the overall survival rate was $54 \%$ with a median survival of 74 months (Figure 2). Univariate analysis showed that age, gender, chronic obstructive pulmonary disease (COPD), coronary artery disease (CAD), obstructive sleep apnea, peripheral vascular disease, steroid use, diabetes mellitus (DM), renal insufficiency, preoperative imaging status, and mediastinal staging met the criteria to be entered into the multivariate analysis. The multivariate analysis showed that the patient's age and peripheral vascular disease were factors that were associated with worse survival in this group (Table 2).

\section{Discussion}

Overall there are three strategies for treating patients with stage IIIA-N2 disease. For patients where the pathologic $\mathrm{N} 2$ disease is discovered after surgery or occult N2 disease, studies show that there is a significant benefit for having

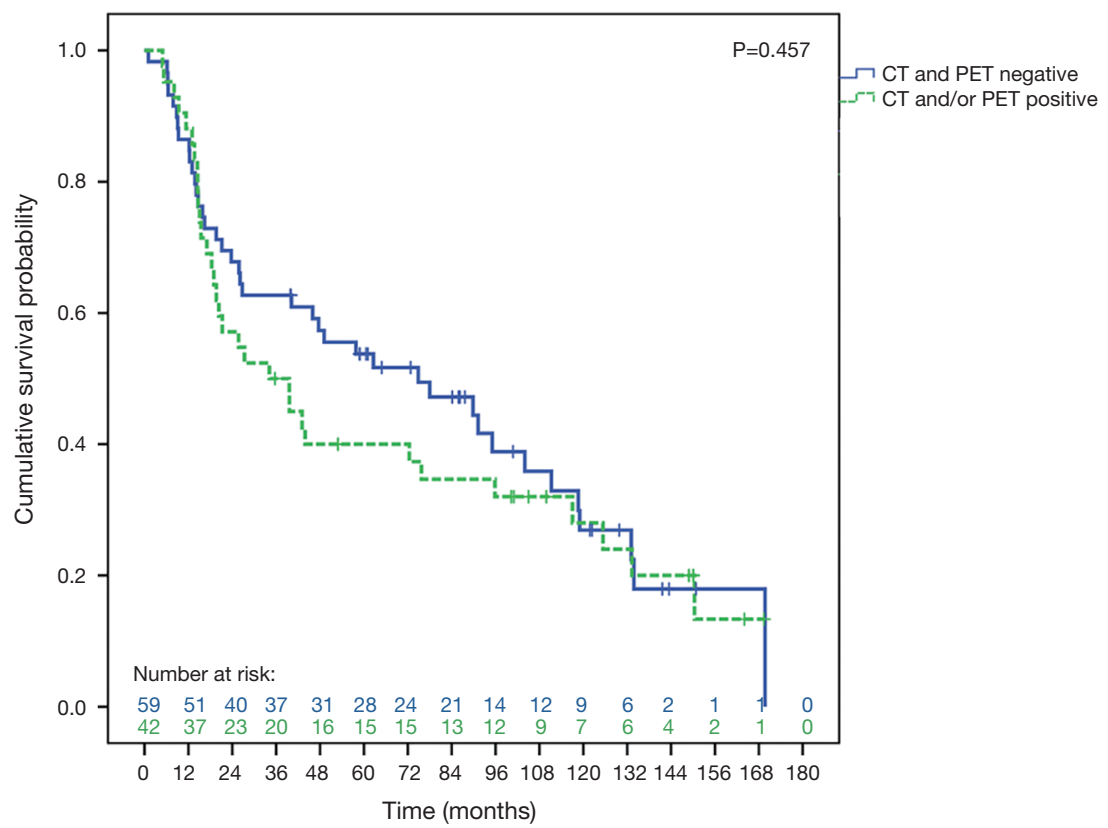

Figure 2 Survival based on preoperative imaging. Kaplan-Meier curve shows that patients with CT and PET negative preoperative imaging has the overall survival rate was $54 \%$ with a median survival of 74 months but there was no significant difference between this group and patients with $\mathrm{CT}$ and/or PET positive preoperative imaging ( $\mathrm{P}=0.457)$. CT, computed tomography; PET. positron emission tomography. 
Table 2 Univariate and multivariate analysis for survival

\begin{tabular}{|c|c|c|c|c|c|}
\hline Characteristics & Frequency & $P$ value & $\mathrm{HR}$ & \multicolumn{2}{|c|}{$95 \% \mathrm{Cl}$ for $\mathrm{HR}$} \\
\hline Age & 101 & 0.014 & 1.036 & 1.007 & 1.066 \\
\hline Peripheral vascular disease & 7 & 0.038 & 2.486 & 1.052 & 5.875 \\
\hline
\end{tabular}

$\mathrm{Cl}$, confidence interval; HR, hazard ratio.

both adjuvant chemotherapy and adjuvant radiation therapy. IALT, ANITA and LACE have shown that stage IIIA-N2 patients can benefit from adjuvant chemotherapy (14-16). The ANITA study showed a 5 -year survival rate of $42 \%$ versus $26 \%$ in stage IIIA (15). Postoperative radiation therapy for patients with completely resected non-small cell lung cancer (NSCLC) was detrimental to patients with early stage lung cancer but it was unclear if it could benefit patients with IIIA-N2 disease (17) Subsequent studies have shown that patients who underwent complete resection with sequential chemotherapy and radiation showed improved overall survival from 45.7 versus 40.7 months (18). Most patients in our study received adjuvant chemoradiation therapy with comparable overall median survival of 49 months.

For those patients who have known N2 disease, the standard therapy has been induction chemotherapy followed by surgery and adjuvant radiation therapy or induction chemoradiation therapy followed by surgery. However, for patients who are found to have N2 disease at the initial surgery it is unknown the role of adjuvant therapy. Most of our patients received adjuvant chemoradiation therapy however, multivariate analysis did not show that adjuvant chemoradiation therapy improved survival.

In the area of improved mediastinal staging, it is unknown if patients with $\mathrm{N} 2$ disease not evident on PET and CT of chest with biopsy proven N2 disease will benefit from induction chemotherapy followed by surgery and radiation therapy compared to surgery first in the multimodality treatment plan. Since most of the patients that were studied in the randomized controlled trial had CT positive lymph nodes, the benefit is not clear in this subset. Although the difference was not statistically significantly from other groups, in our study, the median survival for PET and CT negative N2 disease group of patients was 72 months. Our group may have had less mediastinal lymph node burden compared to other groups of patients since despite invasive mediastinal staging, they were found not to have N2 disease prior to surgery. Most of the patients had small peripheral lung cancer without CT or FDGPET evidence of mediastinal disease, thus they did not undergo mediastinal staging. Those patients who had any CT or FDG-PET evidence or large tumor or central tumor underwent mediastinal staging. If the EBUS was negative but still high clinical suspicion, patient underwent mediastinoscopy. Despite this protocol this select groups of patients did not have N2 disease prior to surgery. However, this protocol has led to only $3.6 \%$ of patients having occult $\mathrm{N} 2$ disease after surgical resection during this time period. Patients with PET N2 positive lymph nodes did not have worse survival in this study nor were patients who had $>1 \mathrm{~cm}$ lymph node on a CT scan of the chest. The location of the N2 disease also did not predicts worse survival.

The overall 5 -year survival for patients with stage IIIA-N2 disease in the seventh edition of AJCC staging for NSCLC has been $24 \%$ (19). Our study shows that our group of patients who were thought to have clinical stage $\mathrm{N} 0$ or N1 disease at the time of surgery and found to have pathologic N2 disease after surgery had excellent overall 5 -year survival of $48 \%$. This is due to heterogeneity of N2 disease where some patients have significant tumor burden in the mediastinal lymph nodes which may have worse overall survival compared to patients with occult disease in the mediastinal lymph nodes (20).

Our retrospective study has several limitations. One limitation is FDG-PET was performed from 2000 to 2004 and FDG-PET/CT was used from 2004 to 2011. We did not have the original scan for CT and PET/CT for all of the patients and we relied on reports for those patients where we did not have imaging information.

Further studies are needed to understand the best way to treat patients with PET and CT negative, occult IIIA-N2 disease. One question is to determine whether patients with $\mathrm{CT}$ and PET negative mediastinum with positive $\mathrm{N} 2$ on EBUS to undergo surgery first strategy in multimodality therapy may have a good overall survival rate compared to 
induction chemotherapy followed by surgery and radiation therapy. A randomized control trial for this group of patients may answer this important question.

\section{Conclusions}

In summary, the patients with clinical N0 or N1 disease who had initial surgery and found to have pathologic N2 disease has an excellent five-year survival rate. This may be due to heterogeneity of $\mathrm{N} 2$ disease and the improved survival for patients with less metastatic disease.

\section{Acknowledgements}

We thank Anna Saikin for language editing of the manuscript.

\section{Footnote}

Conflicts of Interest: MP Kim has consulted for Intuitive Surgical, Olympus, Boston Scientific and Medtronics. The other authors have no conflicts of interest to declare.

Ethical Statement: The University of Texas MD Anderson Cancer Center (MDACC) Institutional Review Board approved this retrospective study (PA15-0619).

\section{References}

1. Rosell R, Gomez-Codina J, Camps C, et al. A randomized trial comparing preoperative chemotherapy plus surgery with surgery alone in patients with non-small-cell lung cancer. N Engl J Med 1994;330:153-8.

2. Roth JA, Fossella F, Komaki R, et al. A randomized trial comparing perioperative chemotherapy and surgery with surgery alone in resectable stage IIIA non-small-cell lung cancer. J Natl Cancer Inst 1994;86:673-80.

3. Roth JA, Atkinson EN, Fossella F, et al. Long-term followup of patients enrolled in a randomized trial comparing perioperative chemotherapy and surgery with surgery alone in resectable stage IIIA non-small-cell lung cancer. Lung Cancer 1998;21:1-6.

4. Rosell R, Gomez-Codina J, Camps C, et al. Preresectional chemotherapy in stage IIIA non-small-cell lung cancer: a 7-year assessment of a randomized controlled trial. Lung Cancer 1999;26:7-14.

5. Depierre A, Milleron B, Moro-Sibilot D, et al. Preoperative chemotherapy followed by surgery compared with primary surgery in resectable stage I (except T1N0),
II, and IIIa non-small-cell lung cancer. J Clin Oncol 2002;20:247-53.

6. Gilligan D, Nicolson M, Smith I, et al. Preoperative chemotherapy in patients with resectable non-small cell lung cancer: results of the MRC LU22/NVALT 2/ EORTC 08012 multicentre randomised trial and update of systematic review. Lancet 2007;369:1929-37.

7. Scagliotti GV, Pastorino U, Vansteenkiste JF, et al. Randomized phase III study of surgery alone or surgery plus preoperative cisplatin and gemcitabine in stages IB to IIIA non-small-cell lung cancer. J Clin Oncol 2012;30:172-8.

8. Felip E, Rosell R, Maestre JA, et al. Preoperative chemotherapy plus surgery versus surgery plus adjuvant chemotherapy versus surgery alone in early-stage nonsmall-cell lung cancer. J Clin Oncol 2010;28:3138-45.

9. Albain KS, Rusch VW, Crowley JJ, et al. Concurrent cisplatin/etoposide plus chest radiotherapy followed by surgery for stages IIIA (N2) and IIIB non-small-cell lung cancer: mature results of Southwest Oncology Group phase II study 8805. J Clin Oncol 1995;13:1880-92.

10. Darling GE, Li F, Patsios D, et al. Neoadjuvant chemoradiation and surgery improves survival outcomes compared with definitive chemoradiation in the treatment of stage IIIA N2 non-small-cell lung cancer. Eur J Cardiothorac Surg 2015;48:684-90; discussion 690.

11. Darling GE, Allen MS, Decker PA, et al. Randomized trial of mediastinal lymph node sampling versus complete lymphadenectomy during pulmonary resection in the patient with $\mathrm{N} 0$ or N1 (less than hilar) non-small cell carcinoma: results of the American College of Surgery Oncology Group Z0030 Trial. J Thorac Cardiovasc Surg 2011;141:662-70.

12. Lee PC, Port JL, Korst RJ, et al. Risk factors for occult mediastinal metastases in clinical stage I non-small cell lung cancer. Ann Thorac Surg 2007;84:177-81.

13. Al-Sarraf N, Aziz R, Gately K, et al. Pattern and predictors of occult mediastinal lymph node involvement in non-small cell lung cancer patients with negative mediastinal uptake on positron emission tomography. Eur J Cardiothorac Surg 2008;33:104-9.

14. Group NM-aC, Arriagada R, Auperin A, et al. Adjuvant chemotherapy, with or without postoperative radiotherapy, in operable non-small-cell lung cancer: two meta-analyses of individual patient data. Lancet 2010;375:1267-77.

15. Douillard JY, Rosell R, De Lena M, et al. Adjuvant vinorelbine plus cisplatin versus observation in patients with completely resected stage IB-IIIA non-small-cell lung cancer (Adjuvant Navelbine International Trialist 
Association [ANITA]): a randomised controlled trial. Lancet Oncol 2006;7:719-27.

16. Pignon JP, Tribodet H, Scagliotti GV, et al. Lung adjuvant cisplatin evaluation: a pooled analysis by the LACE Collaborative Group. J Clin Oncol 2008;26:3552-9.

17. Postoperative radiotherapy in non-small-cell lung cancer: systematic review and meta-analysis of individual patient data from nine randomised controlled trials. PORT Metaanalysis Trialists Group. Lancet 1998;352:257-63.

18. Robinson CG, Patel AP, Bradley JD, et al. Postoperative radiotherapy for pathologic N2 non-small-cell lung cancer

Cite this article as: Kim MP, Correa AM, Hofstetter WL, Mehran RJ, Rice DC, Roth JA, Vaporciyan AA, Walsh GL, Erasmus JJ, Swisher SG. Occult stage IIIA-N2 patients have excellent overall survival with initial surgery. J Thorac Dis 2018;10(12):6670-6676. doi: 10.21037/jtd.2018.10.94 treated with adjuvant chemotherapy: a review of the National Cancer Data Base. J Clin Oncol 2015;33:870-6.

19. Goldstraw P, Crowley J, Chansky K, et al. The IASLC Lung Cancer Staging Project: proposals for the revision of the TNM stage groupings in the forthcoming (seventh) edition of the TNM Classification of malignant tumours. J Thorac Oncol 2007;2:706-14.

20. Rocco G, Nason K, Brunelli A, et al. Management of stage IIIA (N2) non-small cell lung cancer: A transatlantic perspective. J Thorac Cardiovasc Surg 2016;151:1235-8. 